\title{
FISH and Calcofluor staining techniques to detect in situ filamentous fungal biofilms in water
}

\author{
Ana B. Gonçalves, Isabel M. Santos, R. Russell M. Paterson and \\ Nelson Lima \\ Centro de Engenharia Biológica, Micoteca da Universidade do Minho, Braga, Portugal \\ Summary Filamentous fungi are a ubiquitous and diverse group of eukaryotic organisms \\ and may contribute, along with bacteria, yeasts, protozoa and viruses, to the \\ formation of biofilms in water distribution systems. However, fungal involvement \\ in biofilms has not been demonstrated unambiguously. Furthermore, these fungi \\ may be responsible for the production of tastes, odours and mycotoxins in \\ drinking water making their early detection important. To overcome these \\ problems a combination of two fluorescent techniques for direct detection was \\ tested: (a) Fluorescence In Situ Hybridization (FISH) employing the universal \\ rRNA probe EUK516, labelled with the red Cy3, and (b) staining with \\ Calcofluor White MR2 fluorescent dye which stains fungal cell walls blue. \\ Pure cultures of Penicillium brevicompactum were used to establish the \\ methods followed by separate experiments with real water biofilm samples in \\ PVC-C and cast iron coupons. FISH demonstrated eukaryotic microrganisms \\ after approximately $5 \mathrm{~h}$ while the calcofluor method revealed chitinous and/or \\ cellulose containing filamentous structures in less than one hour. When the two \\ methods were combined, additional resolution was obtained from the images of \\ filamentous walls (blue) with intact protoplasm (red). In conclusion, FISH and \\ Calcofluor staining provide rapid, and direct information on the involvement of \\ filamentous fungi in biofilms which form in water.
}

Key words FISH, Calcofluor, Staining techniques, Filamentous fungi, Biofilms, Water

The occurrence of biofilms in potable water distribution systems can be a source of taste, odour and visual appearance problems, resulting in poor drinking water quality [12]. Furthermore, health problems may arise if pathogenic microorganisms develop in the biofilm and are later released into the water flow. A biofilm is a microbial community attached to a surface or interface and embedded in an extracellular polymer matrix which the community of microorganisms have produced. This community can include bacteria, filamentous fungi, yeasts, protozoa and other organisms. Several species of filamentous fungi are known producers of aromas, pigments and mycotoxins,

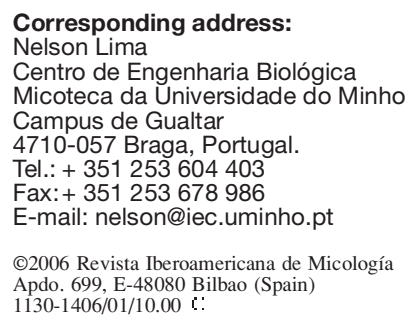

nevertheless the contribution of filamentous fungi to biofilm formation in water distribution systems is still an area of some speculation compared to the more accepted role for bacteria.

Filamentous fungi are excellent colonizers of surfaces and the formation of fungal biofilms was well demonstrated by Elvers et al. [6]. Fungi were probably amongst the groups of organisms detected in the biofilms in drinking water distribution systems [14] but the most convincing evidence is presented in [5]. One abiding problem is to visualise the filamentous fungi as they are present in biofilms. Identification using conventional plating out methodology is indirect and time consuming. Fluorescence stains and transmission electron microscopy were used to determine filamentous fungi cells in endolithic biofilms [4]. In 1989, De Long [3] first used fluorescently labelled oligonucleotides for the detection of single microbial cells. Over the last decade, sensitivity and speed have made FISH a powerful tool for many applications in all fields of microbiology. Aureobasidium pullulans, a yeast-like fungus that grows on living leaves has been studied using FISH [8]. In medicine, Candida spp. are a constant threat for immunocompromised patients, and the yeast was also detected by FISH [10]. Sterflinger and Hain in 1999 [15] developed oligonucleotide probes for the detection of black yeasts and other fungi that were grown on glass and plastic surfaces.

Molecules of rRNA are the main target in the vast majority of FISH applications. The oligonucleotide probes 
used in FISH are generally between 15 and 30 nucleotides long, target the site $16 \mathrm{~S}$ or $18 \mathrm{~S}$ rRNA, which are sub-units of Bacteria and Archae or Eucarya groups respectively, and are covalently linked at the 5'-terminal to a single fluorescent dye molecule. Common fluorochromes include fluorescein, tetramethylrhodamine, texas red and carbocyanine dyes (Cy5 or Cy3). The latter have greatly increased the sensitivity of FISH. In this work the universal EuCarya probe EUK516, targeting nucleotides 502-516 of the 18S rRNA molecule of Saccharomyces cerevisiae [7], labelled with $\mathrm{Cy} 3$, was used.

Another approach is the use of Calcofluor White M2R staining for the visualization of fungal cell walls. The fungal cell wall is a complex structure composed mainly of polysaccharides and their chemical complexes with proteins. The crystalline polysaccharides, chitin and [3-glucans, constitute the skeletal portion of the wall. Calcofluor binds with chitin and cellulose and is used especially in medicine $[2,9]$.

In the present paper, the use of FISH and calcofluor white M2R staining techniques for the detection of filamentous fungi in water biofilms are described, which may provide inherent advantages over conventional plating out methods.

\section{Material and methods}

Samples. Penicillium brevicompactum MUM 05.17 was grown in Eppendorf tubes on $1 \mathrm{ml}$ malt extract broth at $25{ }^{\circ} \mathrm{C}$ for three days. Pure cultures of this fungus were used in this study, to establish the methods. The water biofilm samples in PVC-C and cast iron (CI) coupons, with $3 \mathrm{~cm}$ diameter, were obtained from a potable water up-flow laboratorial reactor.

Oligonucleotide probe and FISH technique. The oligonucleotide probe EUK516, 5'-ACCAGACTTGCCCTCC-3', labelled with the dye $\mathrm{Cy} 3$ was purchased from MWG Biotech (Ebersberg, Germany). The protocol for FISH technique was established with a culture of P. brevicompactum. After the growth of the fungus, $1 \mathrm{~mL}$ of the suspension was centrifuged for $5-10 \mathrm{~min}$ at $12000 \mathrm{rpm}$. The resulting mycelial pellet was resuspended in $750 \mu$ of cold ethanol $(90 \%)$ and stored at room temperature for $10 \mathrm{~min}$. This suspension was then vortexed at full speed for 2 min after which $10 \mu \mathrm{l}$ was spotted onto a microscope slide and air dried. $6 \mathrm{ml}$ of an hybridisation solution (HS: $0.9 \mathrm{M} \mathrm{NaCl}$, $20 \mathrm{mM}$ Tris $/ \mathrm{HCl}, 0.02 \% \mathrm{w} / \mathrm{v}$ SDS, $35 \% \mathrm{v} / \mathrm{v}$ formamide, $\mathrm{pH} 8)$ and $4 \mu \mathrm{l}$ of probe $(5 \mathrm{ng} / \mu \mathrm{l})$ were added. Then the microscope slide was incubated at $46{ }^{\circ} \mathrm{C}$ for $3 \mathrm{~h}$. The excess of probe was removed by incubation for $20 \mathrm{~min}$ at $48{ }^{\circ} \mathrm{C}$ with $2 \mathrm{ml}$ of washing solution (WS: $0.9 \mathrm{M} \mathrm{NaCl}$, $20 \mathrm{mM}$ Tris $/ \mathrm{HCl}, 0.05 \mathrm{M}$ EDTA and $0.02 \% \mathrm{w} / \mathrm{v} \mathrm{SDS}$, $\mathrm{pH}$ 8). Finally, the washing solution was removed with sterile distilled water. The real samples, i.e., coupons, were treated similarly. First the PVC-C or CI coupons were dehydrated for $10 \mathrm{~min}$ in $90 \%(\mathrm{v} / \mathrm{v})$ ethanol. Then, $500 \mathrm{\mu l}$ of the HS with $5 \mathrm{ng} / \mathrm{ul}$ probe was applied to the samples which were incubated for $3 \mathrm{~h}$ at $46^{\circ} \mathrm{C}$. The HS was removed by incubating the samples in $10 \mathrm{ml}$ of WS for $20 \mathrm{~min}$ at $48{ }^{\circ} \mathrm{C}$. Afterwards the samples were also rinsed with distilled water. As negative controls, samples of pure culture were treated as mentioned above but without addition of the probe.

Staining with Calcofluor. The presence of filamentous structures in the biofilms was analysed in situ with Calcofluor White M2R stain. The mycelium of
$P$. brevicompactum previously hybridized with the probe EUK516 was stained with $10 \mu \mathrm{l}$ of $25 \mu \mathrm{M}$ Calcofluor dye solution in the dark at room temperature for $30 \mathrm{~min}$. After staining, $100 \mu \mathrm{l}$ of sterile distilled water was used to wash the excess of Calcofluor and a coverslip was applied for observation under the epifluorescent microscope. In respect to the water biofilms in the coupons, about $300 \mathrm{ml}$ of the dye solution was applied to the surface of the coupons which were incubated in the dark at room temperature for $30 \mathrm{~min}$. After the staining procedure, the samples were washed with $3 \mathrm{ml}$ of sterile distilled water for microscopy.

Microscopy. After FISH and Calcofluor staining, the microscope slide with pure culture and the coupons with biofilm samples were observed under an Axioskop epifluorescent microscope (Carl Zeiss, Germany) using UV light equipped with $40 \mathrm{X} / 0.30$ and $10 \mathrm{X} / 0.65$ objectives.

The excitation wavelength for the Calcofluor stain was $346 \mathrm{~nm}$ and the signal acquired is blue, while for Cy3 the excitation wavelength was $543 \mathrm{~nm}$ and the signal acquired is red. Image processing was carried out with the Zeiss software package. Virtual images, resulting from the overlaping of the two images obtained for each stain, blue or red, were created using the Paint Shop Pro 7 image processing programme (Canada).

\section{Results}

All tests with $P$. brevicompactum, using either the EUK516 probe or the Calcofluor dye, gave a positive result (Table 1) with strong fluorescent signals (Figure 1). The fungal cell walls are visible as blue filaments (Figure 1-A, D) and the Cy3-labelled EUK516 probe applied to the same area, hybridized with the rRNA sequences in this eukaryotic microorganism and stained red (Figure 1-B, E).

The superimposition of the two images obtained with each staining technique (Figure 1-C, F) gives further information enabling the visualization of physiologically active portions of the mycelium in pink, resulting from the combination of empty filaments in blue with intact protoplasm in red.

When these techniques were applied to the water biofilms $76 \%$ of the samples for the PVC-C coupons and $54 \%$ of the samples for the CI coupons showed filamentous growth as detected by calcofluor staining (Table 1). Of the studied coupons, only $24 \%$ of the PVC-C samples and $8 \%$ of the CI samples (Table 1) exhibited a hybridization signal with the EUK516 probe (Figure 2).

In general, the intensity of fluorescence signals obtained for FISH was significantly lower for the biofilm samples than for the $P$. brevicompactum experiments. Therefore, the autofluorescence of the biofilm samples in the Cy3 red channel was investigated (Figure 3). PVC-C coupons exhibited autofluorescence (red) which is accentuated at higher magnifications (Figure 3-A); as a result specific fluorescence signals of the used probe may be masked. The autofluorescence was faint in CI coupons (Figure 3-B).

Table 1. Number of samples analysed with FISH and Calcofluor techniques and percentage of positive results.

\begin{tabular}{lccc}
\hline & No of samples & FISH & Calcofluor \\
\hline Negative control & 2 & 0 & - \\
P. brevicompactum & 6 & $100 \%$ & $100 \%$ \\
PVC-C coupon & 17 & $24 \%$ & $76 \%$ \\
Cl coupon & 13 & $8 \%$ & $54 \%$ \\
\hline
\end{tabular}



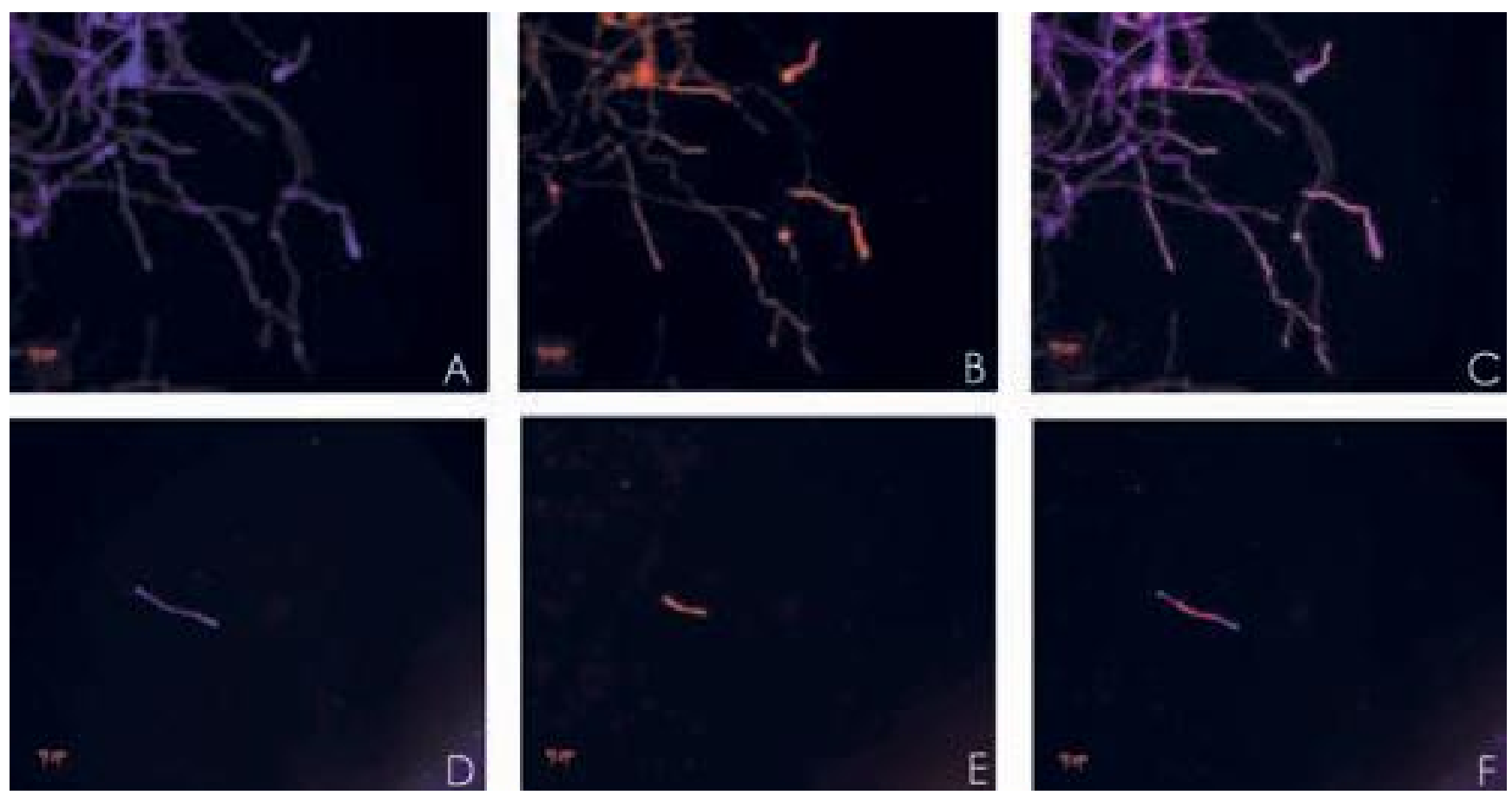

Figure 1. Visualisation of Penicillium brevicompactum. Images A, B, C represent a mass of hyphae and D, E, F represent a single filament. (A, D) Detection of mycelia by Calcofluor staining. (B, E) Detection of the same structures with EUK516 probe. (C, F) Overlap of the two images using an image processing programme.
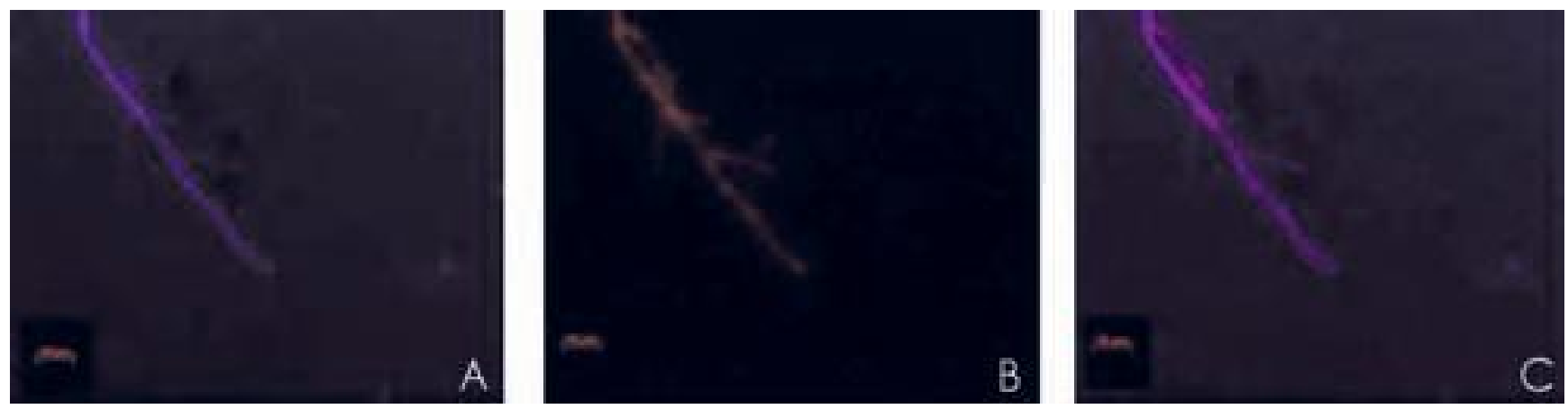

Figure 2. Test with PVC-C coupon. (A) Detection of fungal mycelia by Calcofluor staining. (B) Detection of fungal mycelia using EUK516 probe. (C) Overlap of the two images using an image processing programme.
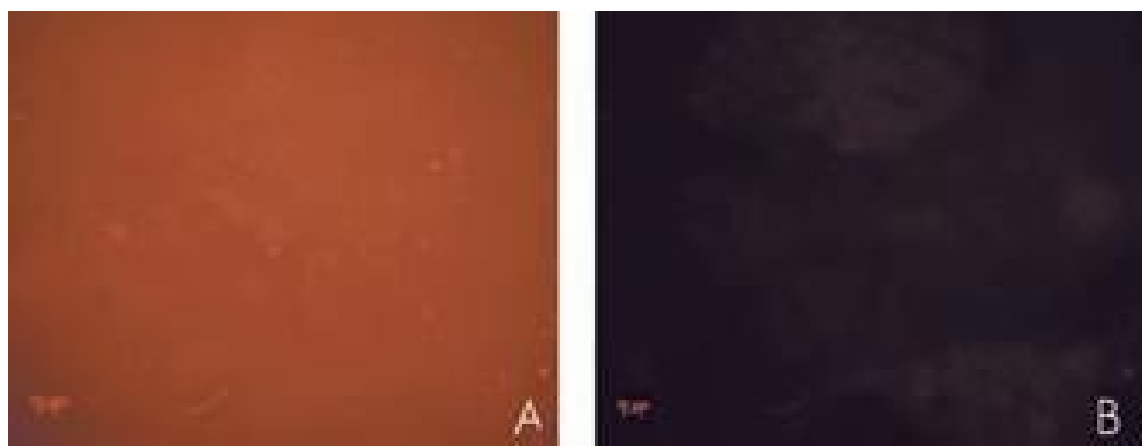

Figure 3. Autofluorescence of different materials in Cy3 channel using 400X of magnification. (A) PVC-C and (B) Cl coupons. 


\section{Discussion}

Calcofluor staining is easy to perform and constitutes an effective and rapid method, taking less than one hour, to detect the presence of presumptive filamentous fungi structures on biofilms of water pipelines.

A typical FISH protocol includes four steps: the fixation and permeabilisation of the sample, hybridisation, washing step to remove unbound probe and detection of labelled cells by microscopy. The procedure can be completed in a few hours. In the present study, $5 \mathrm{~h}$ were sufficient. In situ hybridization with the rRNA EUK516 probe not only gives an indication of the presence of an eukaryotic organism but also information about physiological cell activity. Therefore, low hybridization signals can either reflect the low occurrence of the target organisms or low rRNA content of cells with little activity. On the other hand, although the membranes are expected to be permeable after fixation an additional permeabilisation step can be sometimes necessary to allow the penetration of the probe. Prigione and Marchisio [13] consider this step fundamental to enhance the staining of fungal conidia with fluorescence dyes. Preliminary tests were conducted (data not shown) with an enzymatic treatment in pure culture using a cell wall lysing enzyme from Bacillus subtilis, B-(1-4) glucanase (Fluka, Germany), but signals were not improved so all hybridisations with pure cultures of $P$. brevicompactum or biofilm samples were done without any enzymatic treatment.

A significant proportion of the PVC-C coupon samples and the only positive CI sample gave low hybridization signals with EUK516, where calcofluor staining showed filamentous chitin and/or cellulose structures typical of fungi. In the case of the PVC-C coupons the strong autofluorescence of the material, enhanced by high magnification, may have masked the signal. The corroded surface of CI material also interfered with the visualization of the hybridization signal.

Water biofilms can be viewed as low nutrient, highly competitive environments with small cellular growth rates and little physiological activity. Amann et al. [1] stressed the difficulty of detecting cells in situ in these conditions, with a low rRNA content, using fluorescently labelled rRNA probes. Muller et al. [11] overcame this problem by mixes of several monolabeled oligonucleotides targeted to independent sites on the rRNA molecule with identical specificity, in order to amplify the fluorescence signal. These authors used a confocal laser scanning microscope (CLSM) for in situ analysis of fungal biofilms on historic window glass. CLSM combines the advantages of digital fluorescence and the possibility to detect optical sections of biofilm samples with enhanced vertical and axial resolution and quality.

To our knowledge, one of the few studies where Calcofluor and FISH techniques are applied to fungal populations in environmental samples is that of Tomaszewski et al. [16] although recently more FISH applications to complex samples, such as activated-sludge or soil, have been published.

In conclusion, Calcofluor White M2R staining demonstrates the presence of chitin or cellulose filamentous structures typical of fungi and rRNA in situ hybridisation confirms the presence of eukaryotic. This combined approach is useful to provide evidence that the organisms under study are filamentous fungi.
This work was undertaken as part of a research project supported by the European Union within the Fifth Framework Programme, "Energy, environment and sustainable development programme", $n^{\circ}$ EVK1-2002-00108. There hereby follows a disclaimer stating that the authors are solely responsible for the work, it does not represent the opinion of the community and the community is not responsible for any use that might be made of data appearing herein. R.R.M. Paterson was supported by the grant FRH/BPD/14923/2004 from Fundação para a Ciência e Tecnologia (FCT), Portugal. 


\section{References}

1. Amann R, Fuchs BM, Behrens S. The identification of microorganisms by fluorescence in situ hybridisation. Curr Opin Biotechnol 2001; 12: 231-236.

2. Baselski VS, Robison MK, Pifer LW, Woods DR. Rapid detection of Pneumocystis carinii in bronchoalveolar lavage samples by using Cellufluor staining. J Clin Microbiol 1990; 28: 393-394.

3. De Long EF, Wickham GS, Pace NR Phylogenetic stains: ribosomal RNA-based probes for the identification of single microbial cells. Science 1989; 243: 1360-1363.

4. De los Rios A, Wierzchos J, Sancho LG, Ascaso C. Exploring the physiological state of continental antarctic endolithic FEMS Microbiol Ecol 2004; 50: 143-152.

5. Dogget MS. Characterization of fungal biofilms within a municipal water distribution system. Appl Environ Microbiol 2000; 66: 1249-1251

6. Elvers KT, Leeming K, Lappin-Scott HM. Binary culture biofilm formation by Stenotrophomonas maltophilia and Fusarium oxysorum. J Ind Microbiol Biotechnol 2001; 26: 345-349.
7. Jensen TK, Boye M, Bille-Hansen V. Application of fluorescent in situ hybridisation of Pneumocystis carinii pneumonia in foals and pigs. Vet Pathol 2001; 38: 269-274.

8. Li S, Spear RN, Andrews JH. Quantitative fluorescence in situ hybridisation of Aureobasidium pullulans on microscope slides and leaf surfaces. Appl Environ Microbiol 1997; 63: 3261-3267.

9. Linder M, Winiecka-Krusnell J, Linder E. Use of recombinant cellulose-binding domains of Trichoderma reesei cellulase as a selective immunocytochemical marker for cellulose in Protozoa. Appl Environ Microbiol 2002; 68: 2503-2508.

10. Lischewski A, Kretschmar M, Hof H, Amann R, Hacker J, Morschhauser J. Detection and identification of Candida species in experimentally infected tissue and human blood by rRNA-specific fluorescent in situ hybridisation. J Clin Microbiol 1997; 35: 2943-2948.

11. Muller E, Drewello U, Drewello R, WeiBmann R, Wuertz S. In situ analysis of biofilms on historic window glass using confocal laser scanning microscopy. J Cult Herit 2001; 2: 31-42.
12. Paterson RRM, Lima N. Fungal contamination of drinking water. In: Lehr J, Keeley J, Lehr J, Kingery III TB (Eds.) Control. New Jersey, Hoboken, 2005.

13. Prigione V, Marchisio VF. Methods to maximise the staining of fungal propagules with fluorescent dyes. J Microbiol Methods 2004; 59: 371-379.

14. Sibille I.Biological stability in drinking water distribution systems. L'Année Biologique 1998; 37: 117-161.

15. Sterflinger K, Hain M. In situ hybridisation with rRNA targeted probes as a new tool for the detection of black yeasts and meristematic fungi. Stud Mycol 1999; 43: 23-30.

16. Tomaszewski EK, Logan KS, Snowden KF, Kurtzman CP, Phalen DN. Phylogenetic analysis identifies the "megabacterium" of birds as a novel anamorphic ascomycetous yeast, Macrorhabdus ornithogaster gen. nov., sp.nov. 53: 1201-1205. 